\title{
DUALITY WITH GENERALIZED CONVEXITY
}

\author{
R. R. EGUDO' ${ }^{1}$ AND B. MOND ${ }^{2}$
}

(Received 15 August 1984; revised 25 March 1985)

\begin{abstract}
Recently, Hanson and Mond formulated a type of generalized convexity and used it to establish duality between the nonlinear programming problem and the Wolfe dual. Elsewhere, Mond and Weir gave an alternate dual, different from the Wolfe dual, that allowed the weakening of the convexity requirements. Here we establish duality between the nonlinear programming problem and the Mond-Weir dual using Hanson-Mond generalized convexity conditions.
\end{abstract}

\section{Introduction and preliminaries}

In Hanson and Mond [2], generalized convexity was defined by use of sublinear functionals which satisfy certain convexity type conditions. Wolfe duality was shown to hold under the assumption that a sublinear functional exists such that the Lagrangian satisfies generalized convexity conditions. In [7] Mond and Weir gave a dual for $(\mathrm{P})$, different from the Wolfe dual, where the convexity requirements for duality are considerably weakened. Here we present and prove duality of the Mond-Weir type under the Hanson and Mond [2] generalized convexity conditions,. We also give a strict duality theorem which generalizes the strict duality given in Gulati and Craven [1], Mond and Egudo [6], and Weir [8].

\footnotetext{
${ }^{1}$ Department of Mathematics, La Trobe University, Bundoora, Vic. 3083 Australia. Present address: School of Applied Science, Gippsland Institute of Advanced Education, Churchill, Vic. 3842, Australia.

${ }^{2}$ Department of Mathematics, La Trobe University, Bundoora, Vic. 3083, Australia.

(C) Copyright Australian Mathematical Society 1986, Serial-fee code 0334-2700/86
} 
Definition 1. A functional $F$ is sublinear over a space $S$ if

(A)

(B)

$$
\begin{gathered}
F(x+y) \leqslant F(x)+F(y) \quad \forall x, y \in S \\
F(\alpha x)=\alpha F(x) \text { for } \alpha \in \mathbf{R}, \alpha \geqslant 0, x \in S .
\end{gathered}
$$

From (B) it follows that $F(0)=0$.

DefinIrIon 2. A differentiable function $\phi(x)$ defined on some set $X \subset \mathbf{R}^{n}$ is said to be $F$-convex if

$$
\phi\left(x_{1}\right)-\phi\left(x_{2}\right) \geqslant F_{x_{1}, x_{2}}\left[\nabla \phi\left(x_{2}\right)\right]
$$

$\forall x_{1}, x_{2} \in X$ and for some arbitrary given sublinear functional $F$.

Definition 3. A differentiable function $\phi(x)$ defined on some set $X \subset \mathbf{R}^{n}$ is said to be $F$-quasiconvex if

$$
\phi\left(x_{1}\right) \leqslant \phi\left(x_{2}\right) \Rightarrow F_{x_{1}, x_{2}}\left[\nabla \phi\left(x_{2}\right)\right] \leqslant 0
$$

$\forall x_{1}, x_{2} \in X$ and for some arbitrary given sublinear functional $F$.

Definition 4. A differentiable function $\phi(x)$ defined on a subset $X$ of $\mathbf{R}^{n}$ is said to be $F$-pseudoconvex if

$$
F_{x_{1}, x_{2}}\left[\nabla \phi\left(x_{2}\right)\right] \geqslant 0 \Rightarrow \phi\left(x_{1}\right) \geqslant \phi\left(x_{2}\right)
$$

$\forall x_{1}, x_{2} \in X$ and for some arbitrary given sublinear functional $F$.

Definition 5. A differentiable function $\phi(x)$ defined on $X \subset \mathbf{R}^{n}$ is said to be strictly $F$-pseudo-convex if for $\forall x_{1}, x_{2} \in X, x_{1} \neq x_{2}$,

$$
F_{x_{1}, x_{2}}\left[\nabla \phi\left(x_{2}\right)\right] \geqslant \Rightarrow \phi\left(x_{1}\right) \leqslant \phi\left(x_{2}\right)
$$

for some arbitrary given sublinear functional $F$.

As pointed out in Mond [5], $F$-convex functions can be regarded as a generalization of convex functions. Similarly $F$-pseudoconvex functions and $F$ quasi-convex functions can be regarded as generalizations of pseudoconvex and quasiconvex functions respectively.

Now consider the following pair of nonlinear programming problems:

$$
\text { Minimize } f(x)
$$$$
\text { subject to } g(x) \leqslant 0 \text {; }
$$

(DW) Maximize $f(u)+y^{\prime} g(u)$

$$
\text { subject to } \begin{aligned}
\nabla f(u)+\nabla y^{t} g(u) & =0, \\
y & \geqslant 0 ;
\end{aligned}
$$

where $f: \mathbf{R}^{n} \rightarrow \mathbf{R}, g: \mathbf{R}^{n} \rightarrow \mathbf{R}^{m}$ are differentiable functions.

Let $S$ denote the feasible region of (P) i.e., $S=\{x: g(x) \leqslant 0\}$. 
Hanson and Mond [2] have established the following results.

THEOREM 1 (Weak duality). If for all feasible $x$ in (P) and feasible $(u, y)$ in (DW) there exists a sublinear functional $F$ such that the Lagrangian $f+y^{t} g$ is F-pseudo-convex, then $\operatorname{Min}(\mathrm{P}) \geqslant \operatorname{Max}(\mathrm{DW})$.

THEOREM 2 (Strong duality). If $x^{0}$ is a local or global optimal for $(P)$ and a constraint qualification is satisfied at $x^{0}$, then there exists a $y^{0} \in \mathbf{R}^{m}$ such that $\left(x^{0}, y^{0}\right)$ is feasible in $(D W)$ and the corresponding values of $(\mathrm{P})$ and $(\mathrm{DW})$ are equal. If, also, for all feasible $x$ in $(\mathrm{P})$ and feasible $(u, y)$ in (DW), there exists a sublinear functional $F$ such that $f+y^{t} g$ is $F$-pseudoconvex, then $x^{0}$ and $\left(x^{0}, y^{0}\right)$ are global optima for $(\mathrm{P})$ and $(\mathrm{DW})$ respectively.

\section{Mond-Weir type duality}

We establish duality between (P) and the following Mond-Weir dual (DMW).

(DMW) Maximize $f(u)$

$$
\begin{aligned}
\text { subject to } \nabla f(u)+\nabla y^{t} g(u) & =0, \\
y^{t} g(u) & \geqslant 0, \\
y & \geqslant 0 .
\end{aligned}
$$

TheOREM 3 (Weakly duality). If for all feasible $x$ in (P) and feasible $(u, y)$ in (DMW) there exists a sublinear functional $F$ such that, for feasible $(x, u, y) f$ is F-pseudoconvex and $y^{t} g$ is F-quasiconvex, then Minimum $(P) \geqslant$ Maximum (DMW).

Proof. Since $x$ is feasible for $(\mathrm{P})$ and $(u, y)$ is feasible for (DMW), we have from (5), (9) and (10) that

$$
y^{t} g(x)-y^{t} g(u) \leqslant 0
$$

and, since $y^{t} g$ is $F$-quasiconvex (11) implies

$$
F_{x, u}\left[\nabla y^{t} g(u)\right] \leqslant 0 .
$$

From sublinearity of $F$ we have

$$
F_{x, u}\left[\nabla f(u)+\nabla y^{\prime} g(u)\right] \leqslant F_{x, u}[\nabla f(u)]+F_{x, u}\left[\nabla y^{t} g(u)\right] .
$$

Also from (8) and sublinearity of $F$ we obtain

$$
F_{x, u}\left[\nabla f(u)+\nabla y^{t} g(u)\right]=0 .
$$


Now this and (13) yield

$$
\begin{aligned}
F_{x, u}[\nabla f(u)] & \geqslant-F_{x, u}\left[\nabla y^{\prime} g(u)\right] \\
& \geqslant 0,
\end{aligned}
$$

where (14) follows from (12). Now (14) and $F$-pseudoconvexity of $f$ yields $f(x) \geqslant f(u)$.

THEOREM 4 (Strong duality). Let $x^{0}$ solve (P) and assume a constraint qualification is satisfied at $x^{0}$. Suppose also that a sublinear functional $F$ exists such that $f$ is $F$-pseudoconvex and $y^{\prime} g$ is $F$-quasiconvex for all feasible $x$ in $(\mathrm{P})$ and $(u, y)$ in (DMW). Then there exists a $y^{0}$ such that $\left(x^{0}, y^{0}\right)$ solves (DMW) and Minimum $(P)=$ Maximum $(\mathrm{DMW})$.

Proof. Since $x^{0}$ solves (P) and a constraint qualification is satisfied at $x^{0}$, from Kuhn-Tucker conditions there exists a $y^{0} \in \mathbf{R}_{+}^{m}$ such that $\left(x^{0}, y^{0}\right)$ is feasible for (DMW). Clearly the objective functions of (P) and (DMW) are equal, so the value of $(\mathrm{P})$ equals the value of $(\mathrm{DMW})$ at $x^{0}$. Optimality now follows from weak duality.

We now give an example where Theorems 1 and 2 fail to apply while Theorems 3 and 4 hold.

EXAMPLE 1.

$$
\begin{array}{r}
\text { Minimize } f(x) \equiv x_{1}^{3}-x_{2}^{3}+x_{3}^{3}+x_{3} \\
\text { subject to } g_{1}(x) \equiv x_{1}^{2}-2 x_{1}^{3}+x_{2}^{2}+2 x_{2}^{3}+1 \leqslant 0, \\
g_{2}(x) \equiv-x_{2}^{2}+1 \leqslant 0, \\
g_{3}(x) \equiv-x_{3}+1 \leqslant 0 .
\end{array}
$$

An optimal solution is attained at $x^{0}=(0,-1,1)$. Now $x^{0}=(0,-1,1)$ is feasible for $(\mathrm{P})$ and $\left(u^{0}, y^{0}\right)=(0,0,1,1,1,4)$ is feasible for the Wolfe dual, that is

$$
\begin{gathered}
\text { Maximize } u_{1}^{3}-u_{2}^{3}+u_{3}^{3}+u_{3}+y_{1}\left(u_{1}^{2}-2 u_{1}^{3}+u_{2}^{2}+2 u_{2}^{3}+1\right) \\
+y_{2}\left(1-u_{2}^{2}\right)+y_{3}\left(1-u_{3}\right) \\
\text { subject to }\left[\begin{array}{c}
3 u_{1}^{2} \\
-3 u_{2}^{2} \\
3 u_{3}^{2}+1
\end{array}\right]=-y_{1}\left[\begin{array}{c}
2 u_{1}-6 u_{1}^{2} \\
2 u_{2}+6 u_{2}^{2} \\
0
\end{array}\right]-y_{2}\left[\begin{array}{c}
0 \\
-2 u_{2} \\
0
\end{array}\right]-y_{3}\left[\begin{array}{c}
0 \\
0 \\
-1
\end{array}\right],
\end{gathered}
$$

Now $f(0,-1,1)=3$, but $f\left(u^{0}\right)+y^{0} g\left(u^{0}\right)=4$, where $u^{0}=(0,0,1), y^{0}=(1,1,4)$. Hence weak duality does not hold. Also by considering the third constraint, that is $y_{3}=3 u_{3}^{2}+1$, we find that the objective function tends to infinity as $u_{3}$ tends to minus infinity for any feasible $u_{1}, u_{2}, y_{1}$ and $y_{2}$. Hence strong duality does not hold. 
Examining $f(x) \equiv x_{1}^{3}-x_{2}^{3}+x_{3}^{3}+x_{3}$, we find that $f(x)$ is not pseudo-convex over the feasible region. Hence Mond-Weir results [7] do not apply between Example 1 and its Mond-Weir dual

$$
\begin{gathered}
\text { Maximize } u_{1}^{3}-u_{2}^{3}+u_{3}^{3}+u_{3} \\
\text { subject to }\left[\begin{array}{c}
3 u_{1}^{2} \\
-3 u_{2}^{2} \\
3 u_{3}^{2}+1
\end{array}\right]=-y_{1}\left[\begin{array}{c}
2 u_{1}-6 u_{1} \\
2 u_{2}+6 u_{2}^{2} \\
0
\end{array}\right]-y_{2}\left[\begin{array}{c}
0 \\
-2 u_{2} \\
0
\end{array}\right]-y_{3}\left[\begin{array}{c}
0 \\
0 \\
-1
\end{array}\right], \\
y_{1}\left(u_{1}^{2}-2 u_{1}^{3}+u_{2}^{2}+2 u_{2}^{3}+1\right) \geqslant 0 \\
y_{2}\left(1-u_{2}^{2}\right) \geqslant 0 \\
y_{3}\left(1-u_{3}\right) \geqslant 0 \\
y_{1} \geqslant 0, \quad y_{2} \geqslant 0, \quad y_{3} \geqslant 0
\end{gathered}
$$

However, if we define

$$
F_{x, u}[z]=\left(\sum_{i=1}^{3}\left|z_{i}\right|+a^{t} z\right)\left(f(x)-f(u)+g_{1}(x)+g_{2}(x)\right),
$$

where $a^{t}=(1,-1,1)$ and $x$ is feasible in $(\mathrm{P})$ and $u$ is feasible in (DMW), then

$$
\begin{aligned}
F_{x, u}[\nabla f(u)] & =\left(6\left(u_{1}^{2}+u_{2}^{2}+u_{3}^{2}\right)+2\right)\left(f(x)-f(u)+g_{1}(x)+g_{2}(x)\right) \\
& \leqslant\left(6\left(u_{2}^{1}+u_{2}^{2}+u_{3}^{2}\right)+2\right)(f(x)-f(u)),
\end{aligned}
$$

since $g_{1}(x)$ and $g_{2}(x)$ are non-positive for feasible $x$ in $(\mathrm{P})$. Hence

$$
f(x)-f(u) \geqslant \frac{1}{6\left(u_{1}^{2}+u_{2}^{2}+u_{3}^{2}\right)+2} F_{x, u}[\nabla f(u)] .
$$

So $F_{x, u}[\nabla f(u)] \geqslant 0 \Rightarrow f(x) \geqslant f(u)$.Therefore, $f$ is $F$-pseudoconvex for all feasible $x$ and $u$. Also, since $x$ is feasible for (P) and $(u, y)$ is feasible for (DMW), we have

$$
y^{t} g(x)-y^{t} g(u) \leqslant 0
$$

and

$$
\begin{aligned}
F_{x, u}\left[\nabla y^{t} g(u)\right]= & F_{x, u}[-\nabla f(u)]=F_{x, u}\left[\left(\begin{array}{c}
-3 u_{1}^{2} \\
3 u_{2}^{2} \\
-3 u_{3}^{2}-1
\end{array}\right)\right] \\
= & \left(3 u_{1}^{2}+3 u_{2}^{2}+3 u_{3}^{2}+1-3 u_{1}^{2}-3 u_{2}^{2}-3 u_{3}^{2}-1\right) \\
& \cdot\left(f(x)-f(u)+g_{1}(x)+g_{2}(x)\right) . \\
= & 0 .
\end{aligned}
$$


Hence $y^{t} g$ is $F$-quasiconvex for all feasible $(x, u, y)$. So Theorem 3 is applicable to this pair of nonlinear programs. Now $x^{0}=(0,-1,1)$ solves the primal program and for $\left(x^{0}, y^{0}\right)=\left(0,-1,1, \frac{1}{2}, \frac{1}{2}, 4\right),\left(x^{0}, y^{0}\right)$ is feasible and hence optimal in (DMW) with equality of objective functions.

We now give general results which subsume the different Mond-Weir type duals for the primal problem (PE).

$$
\begin{array}{ll}
\text { Minimize } & f(x), \\
\text { subject to } & g(x) \leqslant 0, \\
& h(x)=0 .
\end{array}
$$

The general Mond-Weir dual to (PE) is (DEG) [7].

$$
\begin{aligned}
\text { Maximize } & f(u)+y_{I_{0}}^{t} g_{I_{0}}(u)+z_{J_{0}}^{t} h_{J_{0}}(u) \\
\text { subject to } \quad \nabla y^{t} g(u)+\nabla z^{t} h(u)+\nabla f(u) & =0, \\
& y_{I_{\alpha}}^{t} g_{I_{\alpha}}(u)+z_{J_{\alpha}}^{t} h_{J_{\alpha}}(u) \\
\alpha & \geqslant 0,2, \ldots, r, \quad y \geqslant 0,
\end{aligned}
$$

where $f: \mathbf{R}^{n} \rightarrow R, g: \mathbf{R}^{n} \rightarrow \mathbf{R}^{m}, h: \mathbf{R}^{n} \rightarrow \mathbf{R}^{k}$ are differentiable functions. $I_{\alpha}, J_{\alpha^{\prime}}$, $\alpha=0,1,2, \ldots, r$ are partitions of the sets $M=\{1,2,3, \ldots, m\}, K=$ $\{1,2,3, \ldots, k\}$ respectively. Also $r=\max \left\{r_{1}, r_{2}\right\}$ where $r_{1}, r_{2}$ is the number of partitions of $M$ and $K$ respectively and $J_{\alpha}=\Phi$ or $I_{\alpha}=\Phi$ for $\alpha>\operatorname{Min}\left\{r_{1}, r_{2}\right\}$. Here $y_{I_{a}}$ denotes the vector consisting of the components $y_{\imath}$ of $y$ such that $i \in I_{\alpha}$. Similar meanings apply to $g_{I_{a}}, z_{J_{\alpha}}$ and $y_{J_{\alpha}}$.

THEOREM 5 (Weak duality). If for all feasible $x$ in (PE) and $(u, y, z)$ in (DEG) there is a sublinear functional $F_{x, u}$ such that $f+y_{I_{0}}^{t} g_{I_{0}}+z_{J_{0}}^{t} h_{J_{0}}$ is $F$-pseudoconvex and $y_{I_{\alpha}}^{t} g_{I_{\alpha}}+z_{J_{\alpha}}^{t} h_{J_{\alpha}}, \alpha=1,2, \ldots, r$ is $F$-quasiconvex, then Minimum (PE) $\geqslant$ Maximum (DEG).

Proof. Since $x$ and $(u, y, z)$ are feasible

$$
y_{I_{\alpha}}^{t} g_{I_{\alpha}}(x)+z_{J_{\alpha}}^{t} h_{J_{\alpha}}(x)-y_{I_{\alpha}}^{t} g_{I_{\alpha}}(u)-z_{J_{\alpha}}^{t} h_{J_{\alpha}}(u) \leqslant 0, \quad \alpha=1,2, \ldots, r,
$$

and since $y_{I_{\alpha}}^{t} g_{I_{a}}+z_{J_{a}}^{t} h_{J_{\alpha}}$ is $F$-quasiconvex, $\alpha=1,2, \ldots, r$, we have

$$
F_{x, u}\left[\nabla\left(y_{I_{\alpha}}^{l} g_{I_{\alpha}}(u)+z_{J_{\alpha}}^{t} h_{J_{\alpha}}(u)\right)\right] \leqslant 0, \quad \alpha=1,2, \ldots, r,
$$

and since $F_{x, u}$ is sublinear we have

$$
\begin{aligned}
F_{x, u}\left[\sum_{\alpha=1}^{r} \nabla\left(y_{l_{\alpha}}^{t} g_{I_{\alpha}}(u)+z_{J_{\alpha}}^{t} h_{J_{\alpha}}(u)\right)\right] & \leqslant \sum_{\alpha=1}^{r} F_{x, u}\left[\nabla\left(y_{I_{\alpha}}^{t} g_{I_{\alpha}}(u)+z_{J_{\alpha}}^{t} h_{J_{\alpha}}(u)\right)\right] \\
& \leqslant 0
\end{aligned}
$$


where (22) follows from (21). Also from the equality constraint of (DEG) i.e. (17) and sublinearity of $F_{x, u}$ we have

$$
\begin{aligned}
& F_{x, u}\left[\nabla\left(f(u)+y_{I_{0}}^{t} g_{J_{0}}(u)+z_{J_{0}}^{t} h_{J_{0}}(u)\right)\right] \\
& \geqslant-F_{x, u}\left[\nabla\left(y_{M \backslash J_{0}}^{t} g_{M \backslash I_{0}}(u)+z_{K \backslash J_{0}}^{t} h_{K \backslash J_{0}}(u)\right)\right] .
\end{aligned}
$$

But $y_{M \backslash I_{0}}^{t} g_{M \backslash I_{0}}+z_{K \backslash J_{0}}^{t}=\sum_{\alpha=1}^{r}\left(y_{I_{\alpha}}^{t} g_{I_{\alpha}}+z_{J_{\alpha}}^{t} h_{J_{\alpha}}\right)$. Therefore (22) and (23) yield

$$
F_{x, u}\left[\nabla\left(f(u)+y_{I_{0}}^{t} g_{I_{0}}(u)+z_{J_{0}}^{t} h_{J_{0}}(u)\right)\right] \geqslant 0
$$

and since $f+y_{I_{0}}^{t} g_{I_{0}}+z_{J_{0}}^{t} h_{J_{0}}$ is $F$-pseudoconvex, then from (24) we have

$$
f(x)+y_{I_{0}}^{t} g_{I_{0}}(x)+z_{J_{0}}^{t} h_{J_{0}}(x) \geqslant f(u)+y_{I_{0}}^{t} g_{I_{0}}(u)+z_{J_{0}}^{t} h_{J_{0}}(u)
$$

and since $y_{I_{0}}^{t} g_{I_{0}}(x)+z_{J_{0}}^{t} h_{J_{0}}(x) \leqslant 0$ we have

$$
f(x) \geqslant f(u)+y_{I_{0}}^{t} g_{I_{0}}(u)+z_{J_{0}}^{t} h_{J_{0}}(u) .
$$

THEOREM 6 (Strong duality). If $x^{0}$ is a local or global minimum of (PE) at which a constraint qualification is satisfied, then there exists $a\left(y^{0}, z^{0}\right)$ such that $\left(x^{0}, y^{0}, z^{0}\right)$ is feasible for (DEG) and the corresponding values of (PE) and (DEG) are equal. If also there exists a sublinear functional $F_{x, u}$ such that $f+y_{I_{0}}^{t} g_{I_{0}}+z_{J_{0}} h_{I_{0}}$ is $F$-pseudoconvex and $y_{I_{\alpha}}^{t} g_{I_{\alpha}}+z_{J_{\alpha}}^{t} h_{J_{\alpha}}, \alpha=1,2, \ldots, r$ is $F$-quasiconvex for all feasible $(x, u, y, z)$, then $x^{0}$ and $\left(x^{0}, y^{0}, z^{0}\right)$ are global optimal solutions of (PE) and (DEG) respectively.

Proor. Since $x^{0}$ solves (PE) either locally or globally and a constraint qualification is satisfied; then by the Kuhn-Tucker conditions [3], [4] there exists a $z^{0} \in \mathbf{R}^{k}$ and $y^{0} \in \mathbf{R}_{+}^{m}$ such that

$$
\begin{aligned}
& \nabla f\left(x^{0}\right)+\nabla y^{0 t} g\left(x^{0}\right)+\nabla z^{0 t} h\left(x^{0}\right)=0, \\
& y^{0} g\left(x^{0}\right)=0, g\left(x^{0}\right) \leqslant 0 \text { and } y^{0} \geqslant 0 .
\end{aligned}
$$

From $g\left(x^{0}\right) \leqslant 0, y^{0} \geqslant 0$, and $y^{0 t} g\left(x^{0}\right)=0$ we conclude that $y_{i}^{0} g_{i}\left(x^{0}\right)=0$ $i=1,2, \ldots, m$ and since $h\left(x^{0}\right)=0$ we must have

$$
y_{I_{\alpha}}^{0 t} g_{I_{\alpha}}\left(x^{0}\right)+z_{J_{\alpha}}^{0 t} h_{J_{\alpha}}\left(x^{0}\right)=0, \quad \alpha=0,1,2, \ldots, r .
$$

Hence $\left(x^{0}, y^{0}, z^{0}\right)$ is feasible in (DEG) and the values of (PE) and (DEG) are equal. Now since there exists a sublinear functional $F_{x, u}$ such that $f+y_{I_{0}}^{t} g_{I_{0}}+$ $z_{J_{0}}^{t} h_{J_{0}}$ is $F$-pseudoconvex and $y_{J_{\mathrm{a}}}^{t} g_{I_{\mathrm{a}}}+z_{J_{\mathrm{a}}}^{t} h_{J_{\alpha}}$ is $F$-quasiconvex $\alpha=1,2, \ldots, r$ for all feasible $(x, u, y, z)$, optimality follows from weak duality.

\section{Converse duality}

Here we give a strict converse duality of Mangasarian type. The result given here generalizes the results obtained by Weir [8], Gulati and Craven [1] and by Mond and Egudo [6]. 
First we consider strict converse duality between (P) and (DMW). Later we shall give a strict converse duality between (PE) and (DEG) which is a general converse dual corresponding to Theorem 6 above.

TheOREM 7 (Strict Converse Duality). Let (P) have an optimal solution at $x^{0}$ at which a constraint qualification is satisfied. Assume that there exists a sublinear functional $F_{x, u}$ such that $f$ is $F$-pseudoconvex and $y^{t} g$ is $F$-quasiconvex for all feasible $x$ in (P) and $(u, y)$ in (DMW). If $(\bar{u}, \bar{y})$ is an optimal solution of (DMW) and $f$ is strictly $F$-pseudoconvex at $\bar{u}$ for all feasible $x$ in $(\mathrm{P})$, then $\bar{u}=x^{0}$ i.e. $\bar{u}$ solves $(\mathrm{P})$.

Proof. We assume $x^{0} \neq \overline{\mathrm{u}}$ and exhibit a contradiction.

Since $x^{0}$ is an optimal solution at which a constraint qualification is satisfied, it follows from Theorem 4 that there exists a $\hat{y}$ such that $\left(x^{0}, \hat{y}\right)$ is an optimal solution for the dual (DMW). Since $(\bar{u}, \bar{y})$ is also optimal for (DMW), it follows that

$$
f\left(x^{0}\right)=f(\bar{u}),
$$

For all feasible $x$ and $(u, y)$, we have $y^{\prime} g(x)-y^{\prime} g(u) \leqslant 0$ and, by $F$-quasiconvexity of $y^{\prime} g$, we have

$$
F_{x, u}\left[\nabla y^{t} g(u)\right] \leqslant 0 .
$$

By (8) and sublinearity of $F_{x, u}$, we have

$$
F_{x, u}[\nabla f(u)] \geqslant-F_{x, u}\left[\nabla y^{t} g(u)\right] .
$$

Now (26) and (27) yield

$$
F_{x, u}[\nabla f(u)] \geqslant 0 .
$$

From (28) and strict $F$-pseudoconvexity of $F_{x, u}$ at $\bar{u}$ we obtain

$$
F_{x, \bar{u}}[\nabla f(\bar{u})] \geqslant 0 \Rightarrow f(x)>f(\bar{u}) \text { for all feasible } x \text { in }(\mathrm{P}) .
$$

And since $x^{0}$ is feasible in (P), we now have $f\left(x^{0}\right) \geqslant f(\bar{u})$ contradicting (25). Hence $x^{0}=\bar{u}$.

TheORem 8 (Strict Converse Duality). Let $x^{0}$ be an optimal solution of (PE) at which a constraint qualification is satisfied. Suppose there exists a sublinear functional $F_{x, u}$ such that $f+y_{I_{0}}^{t} g_{I_{0}}+z_{J_{0}}^{t} h_{J_{0}}$ is F-pseudoconvex and $y_{I_{\alpha}}^{t} g_{I_{a}}+z_{J_{a}}^{t} h_{J_{\alpha}}$ $(\alpha=1,2, \ldots, r)$ are $F$-quasiconvex for all feasible $x$ in $(\mathrm{P})$ and $(u, y, z)$ in (DEG). If $(\bar{u}, \bar{y}, \bar{z})$ is an optimal solution of (DEG) and if also $f+y_{I_{0}}^{t} g_{I_{0}}+z_{J_{0}}^{t} h_{J_{0}}$ is strictly $F$-pseudoconvex at $\bar{u}$, then $\bar{u}=x^{0}$ i.e. $\bar{u}$ solves (PE) and

$$
f\left(x^{0}\right)=f(\bar{u})+\bar{y}_{I_{0}}^{t} g_{I_{0}}(\bar{u})+\bar{z}_{J_{0}}^{t} h_{J_{0}}(\bar{u}) .
$$


Proof. We assume $x^{0} \neq \bar{u}$ and exhibit a contradiction. Since $x^{0}$ is a solution of (PE) at which a constraint qualification is satisfied, it follows from Theorem 6 that there exists $\left(y^{0}, z^{0}\right)$ such that $\left(x^{0}, y^{0}, z^{0}\right)$ solves (DEG). Hence

$$
\begin{aligned}
f\left(x^{0}\right) & =f\left(x^{0}\right)+y_{I_{0}}^{0 t} g_{I_{0}}\left(x^{0}\right)+z_{J_{0}}^{0 t} h_{J_{0}}\left(x^{0}\right) \\
& =f(\bar{u})+\bar{y}_{I_{0}}^{t} g_{I_{0}}(\bar{u})+\bar{z}_{J_{0}}^{t} h_{J_{0}}(\bar{u}) .
\end{aligned}
$$

Now $(\bar{u}, \bar{y}, \bar{z})$ feasible in (DEG) implies

$$
\bar{y}_{I_{\alpha}}^{t} g_{I_{\alpha}}\left(x^{0}\right)+\bar{z}_{J_{\alpha}}^{t} h_{J_{\alpha}}\left(x^{0}\right)-\bar{y}_{I_{\alpha}}^{t} g_{I_{\alpha}}(\bar{u})-\bar{z}_{J_{\alpha}}^{t} h_{J_{\alpha}}(\bar{u}) \leqslant 0, \quad \alpha=1,2, \ldots, r .
$$

This with $F$-quasiconvexity of $y_{I_{\alpha}}^{t} g_{I_{\alpha}}+z_{J_{\alpha}}^{t} h_{J_{\alpha}}, \alpha=1,2, \ldots, r$ for all feasible $(x, u, y, z)$ yields

$$
F_{x, \bar{u}}\left[\nabla\left(\bar{y}_{I_{\alpha}}^{t} g_{I_{\alpha}}(\bar{u})+\bar{z}_{J_{\alpha}}^{t} h_{J_{\alpha}}(\bar{u})\right)\right] \leqslant 0, \quad \alpha=1,2, \ldots, r,
$$

From sublinearity of $F_{x, \bar{u}}$ we have

$$
\begin{aligned}
F_{x, \bar{u}}\left[\sum_{\alpha=1}^{r} \nabla\left(\bar{y}_{I_{\alpha}}^{t} g_{I_{\alpha}}(\bar{u})+\bar{z}_{J_{\alpha}}^{t} h_{J_{\alpha}}(\bar{u})\right)\right] & \leqslant \sum_{\alpha=1}^{r} F_{x, \bar{u}}\left[\nabla\left(\bar{y}_{I_{\alpha}}^{t} g_{I_{\alpha}}(\bar{u})+\bar{z}_{J_{\alpha}}^{t} h_{J_{\alpha}}(\bar{u})\right)\right] \\
& \leqslant 0
\end{aligned}
$$

where (31) follows from (30). From equality constraint of (DEG), that is (17), and sublinearity of $F_{x, \bar{u}}$, we have

$$
\begin{aligned}
F_{x, \bar{u}}\left[\nabla\left(f(\bar{u})+\bar{y}_{I_{0}}^{t} g_{I_{0}}(\bar{u})+\bar{z}_{J_{0}}^{t} h_{J_{0}}(\bar{u})\right)\right] & \geqslant-\sum_{\alpha=1}^{r} F_{x, \bar{u}}\left[\nabla\left(\bar{y}_{I_{\alpha}}^{t} g_{I_{\alpha}}(\bar{u})+\bar{z}_{J_{\alpha}}^{t} h_{J_{\alpha}}(\bar{u})\right)\right] \\
& \geqslant 0,
\end{aligned}
$$

where (32) follows from (31). Now from (32) and strict $F$-pseudoconvexity of $f+\bar{y}_{I_{0}} g_{I_{0}}+\bar{z}_{J_{0}}^{t} h_{J_{0}}$ at $\bar{u}$ we obtain

$$
f(x)+\bar{y}_{I_{0}}^{t} g_{I_{0}}(x)+\bar{z}_{J_{0}}^{t} h_{J_{0}}(x)>f(\bar{u})+\bar{y}_{I_{0}}^{t} g_{I_{0}}(\bar{u})+\bar{z}_{J_{0}}^{t} h_{J_{0}}(\bar{u})
$$

for all feasible $x$ in (PE). From this we obtain

$$
f\left(x^{0}\right)+\bar{y}_{I_{0}}^{t} g_{I_{0}}\left(x^{0}\right)+\bar{z}_{J_{0}}^{t} h_{J_{0}}\left(x^{0}\right)>f(\bar{u})+\bar{y}_{I_{0}}^{t} g_{I_{0}}(\bar{u})+\bar{z}_{J_{0}}^{t} h_{J_{0}}(\bar{u}) .
$$

But $\bar{y}_{l_{0}}^{t} g_{I_{0}}\left(x^{0}\right)+\bar{z}_{J_{0}}^{t} h_{J_{0}}\left(x^{0}\right) \leqslant 0$, hence

$$
f\left(x^{0}\right)>f(\bar{u})+\bar{y}_{I_{0}}^{t} g_{I_{0}}(\bar{u})+\bar{z}_{J_{0}}^{t} h_{J_{0}}(\bar{u})
$$

contradicting (29). So $x^{0}=\bar{u}$.

\section{Some special cases}

We now consider some special cases of the dual (DEG) and Theorems 5, 6 and 8.

(i) If $K=\Phi, I_{0}=M$ then (PE) becomes (P) and (DEG) becomes (DW) and Theorems 5 and 6 reduce to Theorems 1 and 2 respectively. 
(ii) If $K=\Phi, I_{0}=\Phi, I_{1}=M$ then (PE) becomes (P) and (DEG) becomes (DMW) and Theorems 5, 6 and 8 reduce to Theorems 3, 4 and 7 respectively.

(iii) If $I_{0}=M$ and $J_{0}=K$ then (DEG) becomes

(DE)

$$
\begin{array}{ll}
\text { Maximize } & f(u)+y^{t} g(u)+z^{t} h(u) \\
\text { subject to } & \nabla f(u)+\nabla y^{t} g(u)+\nabla z^{t} h(u)=0,
\end{array}
$$

$$
y \geqslant 0 \text {. }
$$

Duality holds between (PE) and (DE) if there exists a sublinear functional $F_{x, u}$ such that $f+y^{t} g+z^{t} h$ is $F$-pseudoconvex.

(iv) If $I_{0}=\Phi, J_{0}=\Phi, I_{1}=M, J_{1}=K$ then (DEG) becomes

Maximize $f(u)$

(DEM)

$$
\text { subject to } \quad \begin{aligned}
\nabla f(u)+\nabla y^{t} g(u)+\nabla z^{t} h(u) & =0, \\
y^{\prime} g(u)+z^{t} h(u) & \geqslant 0, \\
y & \geqslant 0 .
\end{aligned}
$$

If there exists a sublinear functional $F_{x, u}$ such that $f$ is $F$-pseudoconvex and $y^{t} g+z^{t} h$ is $F$-quasiconvex for all feasible $(x, u, y, z)$ then from Theorems 5, 6 and 8 , (DEM) is dual to (PE).

(v) If $I_{0}=\Phi, J_{0}=K, I_{1}=M$ then (DEG) becomes

Maximize $f(u)+z^{\prime} h(u)$

(DMT)

subject to $\nabla f(u)+\nabla y^{t} g(u)+\nabla z^{t} h(u)=0$,

$$
\begin{aligned}
y^{t} g(u) & \geqslant 0, \\
y & \geqslant 0 .
\end{aligned}
$$

If there exists a sublinear functional $F_{x, u}$ such that $f+z^{t} h$ is $F$-pseudoconvex and $y^{\prime} g$ is $F$-quasiconvex, then from Theorems 5, 6 and 8 (DMT) is dual to (PE).

(vi) If $J_{0}=\Phi, I_{0}=M, J_{1}=K$ then (DEG) becomes

(DME)

$$
\text { Maximize } f(u)+y^{t} g(u)
$$$$
\text { subject to } \quad \nabla f(u)+\nabla y^{t} g(u)+\nabla z^{t} h(u)=0,
$$

$$
\begin{aligned}
z^{t} h(u) & \geqslant 0, \\
y & \geqslant 0 .
\end{aligned}
$$

(DME) is dual to (PE) if there exists a sublinear functional $F_{x, u}$ such that $f+y^{t} g$ is $F$-pseudoconvex and $z^{t} h$ is $F$-quasiconvex.

(vii) If $K=\Phi, I_{0}=\Phi, I_{\alpha}=\{\alpha\}, \alpha=1,2, \ldots, m$ then (PE) becomes (P) and

(DEG) becomes

$$
\text { Maximize } f(u)
$$

(DG)

$$
\text { subject to } \quad \begin{aligned}
\nabla f(u)+\nabla y^{\prime} g(u) & =0, \\
y_{\imath} g_{\imath}(u) & \geqslant 0 \quad(i=1,2, \ldots, m), \\
y & \geqslant 0 .
\end{aligned}
$$


If there exists a sublinear functional $F_{x, u}$ such that $f$ is $F$-pseudoconvex and $g$ is $F$-quasiconvex then from Theorems 5, 6 and 8 (DG) is dual to (P).

(viii) In (ii) $y^{t} g$ is required to be $F$-quasiconvex while in (vii) only $g$ is required to be $F$-quasiconvex. It may be possible to combine some but not all components of $g$ into a single $F$-quasiconvex function while the other components are individually but not collectively $F$-quasiconvex. In this case we can find a dual between (DMW) and (DG) as follows. Put $K=\Phi, I_{0}=\Phi$ and $Q \subset M$ then (DEG) becomes

Maximize $f(u)$

subject to $\nabla f(u)+\nabla y^{t} g(u)=0$,

(DM)

$$
\begin{aligned}
\sum_{i \in Q} y_{i} g_{\imath}(u) & \geqslant 0, \\
y_{j} g_{j}(u) & \geqslant 0, \quad j \in M \backslash Q, \\
y & \geqslant 0 .
\end{aligned}
$$

If there exists a sublinear functional $F_{x, u}$ such that $f$ is $F$-pseudoconvex and $\sum_{r \in Q} y_{l} g_{l}$ is $F$-quasiconvex and $g_{l}, j \in M \backslash Q$ are $F$-quasiconvex, then from Theorems 5, 6 and 8, (DM) is dual to (P).

(ix) If only part of the Lagrangian is pseudoconvex, then it is possible to obtain a dual between the Wolfe dual and (DG). For example if $K=\Phi$, and $I_{0}, Q \subset M, I_{0} \cap Q=\Phi$, then (DEG) becomes

$$
\begin{aligned}
\text { Maximize } \quad f(u)+y_{I_{0}}^{t} g_{I_{0}}(u) & \\
\text { subject to } \quad \nabla f(u)+\nabla y^{t} g(u) & =0, \\
\sum_{i \in Q} y_{\imath} g_{\imath} & \geqslant 0, \\
y_{j} g_{j}(u) & \geqslant 0 \quad\left(j \in M \backslash\left(Q \cup I_{0}\right)\right), \\
y & \geqslant 0 .
\end{aligned}
$$

(DWG)

If there exists a sublinear functional $F_{x, u}$ such that $f+y_{I_{0}}^{t} g_{I_{0}}$ is $F$-pseudoconvex and $\sum_{i \in Q} y_{i} g_{i}$ is $F$-quasiconvex and $g_{j}, j \in M \backslash\left(Q \cup I_{0}\right)$ is $F$-quasiconvex for all feasible $(x, u, y)$ then from Theorems 5, 6 and 8, (DWG) is dual to (P).

\section{References}

[1] T. R. Gulati, and B. D. Craven, "A strict converse duality theorem in nonlinear programming," J. Inform. Optim. Sci. 4 (1983), 301-306.

[2] M. A. Hanson and B. Mond, "Further generalizations of convexity in mathematical program. ming," J. Inform. Optim. Sci. 3 (1982), 25-32.

[3] H. W. Kuhn and A. W. Tucker, "Nonlinear programming," Prov. 2 nd Berkeley Symposium on Mathematical Statistics and Probability, (University of California Press 1951), 481-492. 
[4] O. L. Mangasarian, Nonlinear programming (McGraw-Hill, New York, London, Sydney, 1969).

[5] B. Mond, "Generalized convexity in mathematical programming," Bull. Austral. Math. Soc. 27 (1983), 185-202.

[6] B. Mond and R. R. Egudo, "On strict converse duality in nonlinear programming," $J$. Inform. Optim. Sci. 6 (1985), 113-16.

[7] B. Mond and T. Weir, "Generalized concavity and duality," in Generalized concavity in optimization and economics (eds. S. Schaible and W. T. Ziemba), (Academic Press 1981), 263-279.

[8] T. Weir, "Generalized convexity and duality in mathematical programming," Ph.D. thesis, La Trobe University, Bundoora, Melbourne, Australia, 1982,

[9] P. Wolfe, "A duality theorem for nonlinear programming," Quart. Appl. Math. 19 (1961), 239-244. 